\title{
SIGNOS Y SÍNTOMAS DE ADICCIÓN AL CIBERSEXO EN ADULTOS MAYORES
}

\author{
Castro-Calvo, J. \\ Dpto. de Personalidad, Evaluación y Tratamientos Psicológicos \\ Universitat de València, Spain. \\ jesus.castro@uv.es \\ Gil-Juliá, B. \\ Dpto. de Personalidad, Evaluación y Tratamientos Psicológicos \\ Universitat de València, Spain. \\ Enrique-Nebot, J.E. \\ Dpto. de Psicología Básica, Clínica y Psicobiología \\ Universitat Jaume I, Spain \\ Cervigón-Carrasco, $\mathbf{v}$. \\ Dpto. de Psicología Básica, Clínica y Psicobiología \\ Universitat Jaume I, Spain \\ Ballester-Arnal, R. \\ Dpto. de Psicología Básica, Clínica y Psicobiología \\ Universitat Jaume I, Spain
}

Fecha de Recepción: 14 Febrero 2019

Fecha de Admisión: 30 Abril 2019

\section{RESUMEN}

El uso de Internet con objetivos sexuales resulta una práctica cada vez más generalizada en cualquier etapa vital. Si bien en la mayoría de casos el consumo se realiza de forma recreativa, en ocasiones éste puede sobrevenir compulsivo, incontrolado y asociado a un deterioro funcional en diversos ámbitos (adicción al cibersexo). Conocer los síntomas que caracterizan este cuadro clínico constituye una prioridad; sin embargo, disponemos de un pobre conocimiento acerca de su expresión en adultos mayores. Así, este trabajo se planteó con un doble objetivo: 1) analizar la prevalencia de adultos mayores en riesgo de desarrollar o que muestran un perfil patológico de uso del cibersexo y 2) elaborar un perfil de signos y síntomas que lo caracterizan en esta población. Con este objetivo, 538 personas $(77 \%$ hombres) mayores de 60 años $(M=65.3)$ completaron a través de Internet una serie de escalas de conducta sexual online. El 73.2\% afirmaba utilizar internet con objetivo sexual. Entre ellos, el $80.4 \%$ lo hacía de forma recreativa y el $19.6 \%$ restante mostró un consumo de riesgo. Entre los principales síntomas, destacó la percepción de interferencia (presente en el $50 \%$ de los pacientes), pasar $>5$ horas semanales en Internet con fin sexual $(50 \%)$, reconocer que pueden estar haciéndolo excesivamente $(51 \%)$ o la presencia de síntomas de abstinencia (ansiedad, 


\section{SIGNOS Y SÍNTOMAS DE ADICCIÓN AL CIBERSEXO EN ADULTOS MAYORES}

irritabilidad, depresión, etc.) cuando no pueden acceder a contenidos sexuales (24\%). Así, este trabajo destaca la necesidad de visibilizar la actividad sexual online de riesgo en un colectivo silente y normalmente al margen de cualquier intervención para la promoción de la salud sexual online.

Palabras clave: adicción al cibersexo; prevalencia; signos y síntomas; adultos mayores

\section{ABSTRACT}

Signs and symptoms of cybersex addiction in older adults. The use of the Internet for sexual purposes is an increasingly widespread practice at any stage of life. Although in most cases, consumption is done recreationally, sometimes it may become compulsive, uncontrolled, and associated with functional impairment in various areas (addiction to cybersex). Knowing symptoms that characterize this clinical condition is a priority; however, we have poor knowledge about its expression in older adults. Thus, the aim of this work was double: 1) to analyze the prevalence of older adults at risk of developing or showing a pathological profile of cybersex use and 2) to develop a profile of signs and symptoms that characterize it in this population. 538 participants $(77 \%$ men) over 60 years of age $(M=65.3)$ completed a series of online sexual behavior scales. $73.2 \%$ said they used the Internet with sexual aim. Among them, $80.4 \%$ did it recreationally whereas a $20 \%$ showed a risk consumption. Among the main symptoms, the most prevalent were the perception of interference ( $50 \%$ of participants), spending $>5$ hours a week on the Internet for sexual purposes $(50 \%)$, recognize that they may be doing it excessively $(51 \%)$ or presence of symptoms of withdrawal (anxiety, irritability, depression, etc.) (24\%). This work highlights the relevance of visualizing online risky sexual activity in a silent group and usually outside any intervention for the promotion of online sexual health.

Keywords: cybersex addiction; prevalence; signs and symptoms; older adults

\section{INTRODUCCIÓN}

El cibersexo, definido como «el uso de Internet con objetivos de gratificación sexual» (Cooper \& Griffin-Shelley, 2002), comprende actividades que se pueden realizar o bien en solitario o mediante la interacción con otros usuarios online (Shaughnessy, Byers, \& Walsh, 2011). Las más frecuentes serían las que no requieren del contacto otros usuarios (en concreto, la descarga y el visionado de pornografía). Su prevalencia depende de aspectos como el sexo y la edad. En función del sexo, el consumo de pornografía y de cualquier otra actividad sexual online es sistemáticamente menor en las mujeres (Ballester-Arnal, Castro-Calvo, Gil-Llario, \& Giménez-García, 2014). Respecto a su evolución durante el ciclo vital, hasta hace no mucho tiempo el consumo de pornografía resultaba infrecuente hasta alrededor de los 15 años (Wolak, Mitchell, \& Finkelhor, 2007), momento a partir del cual se producía un incremento exponencial que situaba su prevalencia entre el 33-90\% en jóvenes y adultos (Wéry \& Billieux, 2017). Estas estadísticas sin embargo resultarían difícilmente generalizables en el contexto español actual, ya que el contexto cultural y la velocidad con la que los datos quedan obsoletos (debido en parte a la rápida evolución de los contextos digitales) determina en gran medida estas prevalencias. Así, un estudio realizado en España con datos del 2011 documentaba que el porcentaje de jóvenes entre los 18 y los 25 años que afirmaba haber buscado pornografía era del $59 \%$ en chicos y del $24.2 \%$ en chicas (Ballester-Arnal y cols. 2016). En un estudio posterior en el que estos autores analizaban el consumo de pornografía en una base de datos recopilada tan sólo 5 años más tarde, los resultados varían significativamente: a diferencia del estudio antes mencionado, donde el consumo entre los menores de edad resultaba infrecuente, en este estudio en torno al $87 \%$ de los menores de 18 años había consumido porno; asimismo, la prevalencia entre los 18 y 25 años se situaba ya en torno al $98 \%$ en chicos y el $78 \%$ en chicas (lo que supondría un incremento de 28 y 53 puntos porcentuales respecto a estadísticas anteriores) 
(Castro-Calvo, Ballester-Arnal, Gil-Llario, Giménez-García, \& Billieux, 2018). Esta no es la única tendencia documentada: en un trabajo publicado con una muestra de 423 personas entre 60-70 años, el porcentaje de los que había consumido pornografía en alguna ocasión se situaba ya en el 88\% (Castro-Calvo, Ballester-Arnal, Giménez-García, \& Gil-Julià, 2017). Es decir, que prácticamente cualquier persona, independientemente de su sexo o su edad, ha consumido de forma voluntaria pornografía al menos en alguna ocasión.

Otra actividad sexual online relativamente frecuente consistiría en mantener contactos sexuales con otros usuarios (ya sea mediante chat, webcam o intercambio de imágenes). Su prevalencia oscilaría entre el $9.4-30 \%$ en hombres y el $14.9-34 \%$ en mujeres, si bien estos datos probablemente hayan quedado nuevamente obsoletos (Daneback, Cooper, \& Månsson, 2005; Goodson, McCormick, \& Evans, 2001; Shaughnessy et al., 2011). La etapa donde el contacto sexual con otros usuarios resultaría más frecuente sería entre los 18 y 24 años, decreciendo pasada esta franja (Daneback et al., 2005). La actividad más frecuente era el contacto sexual online con una pareja (82.4\%), con conocidos con quien no se mantenía una relación estable $(45.8 \%)$ y con desconocidos (37.1\%) (Shaughnessy \& Byers, 2014). Ateniéndonos a los datos de prevalencias más recientes en nuestro país (en este caso, obtenidos a partir de un muestreo realizado en el año 2011), la prevalencia de este comportamiento se situaba en el $20.5 \%$ en hombres entre $18-25$ años y en $8.6 \%$ en mujeres (Ballester-Arnal, Castro-Calvo, Gil-Llario, et al., 2016). No contamos con datos actuales para jóvenes pero en el estudio mencionado anteriormente con personas mayores de 60 años, la prevalencia de este comportamiento se situaba ya en el $29.4 \%$ en hombres y el $33.3 \%$ en mujeres (Castro-Calvo et al., 2017); sabiendo que el consumo de cibersexo entre las personas de mayor edad tiende a decaer respecto a etapas vitales anteriores, sería presumible que la prevalencia de esta conducta sexual entre personas de menor edad sea incluso mayor, lo que nuevamente confirmaría que se está produciendo un incremento exponencial de este tipo de comportamientos.

En la mayoría de los casos, la principal motivación para recurrir al sexo por Internet es la búsqueda del placer sexual (Castro-Calvo, Giménez-García, Gil-Llario, \& Ballester-Arnal, 2018). Una investigación entre consumidores de cibersexo halló que la mayoría afirmaba que su actividad online satisfacía sus necesidades sexuales entre moderada y completamente (80\% en chicos y $73 \%$ en chicas) (Daneback, Sevcikova, Mänsson, \& Ross, 2013). Otro beneficio del cibersexo lo constituye su potencial educativo: muchos jóvenes -y también adultos- encontrarían en Internet una herramienta útil para suplir la falta de conocimientos sobre sexo o "aprender" mediante la pornografía cómo satisfacer a sus parejas (p.e., descubriendo nuevas posturas sexuales) (Smith, 2013). Sin embargo, no todos valorarían sus beneficios y existe debate en torno a sus consecuencias.

Uno de los principales riesgos del sexo en Internet sería la posibilidad de desarrollar un cuadro clínico de adicción al cibersexo. La adicción al cibersexo se define como el «uso de cibersexo excesivo e incontrolado que desemboca en problemas laborales, sociales y personales» (Cooper \& Griffin-Shelley, 2002). Según Cooper (1998), los pacientes con una adicción al cibersexo invertirían una cantidad de tiempo exagerada realizando la actividad sexual online, persistirían a pesar de las consecuencias negativas, no tendrían control sobre su inicio o su finalización, negarían su problema y fallarían en los intentos por controlarlo. Estos síntomas coincidirían con la reciente propuesta del «trastorno por comportamiento sexual compulsivo», incluido en la reciente revisión de la Clasificación Internacional de Enfermedades de la Organización Mundial de la Salud (CIE-11, Kraus et al., 2018). De acuerdo con Kafka (2013), otro de los síntomas del cuadro clínico sería el uso del cibersexo como regulador de estados emocionales negativos (principalmente ansiedad y depresión). Asimismo, se ha propuesto que alguno de los síntomas utilizados clásicamente para definir las adicciones tóxicas como la tolerancia (aumento de tiempo e intensidad de la actividad online para 


\section{SIGNOS Y SÍNTOMAS DE ADICCIÓN AL CIBERSEXO EN ADULTOS MAYORES}

lograr los efectos iniciales) o bien la abstinencia (aparición de reacciones emocionales intensas tras un tiempo sin cibersexo) serían definitorias de las adicciones sexuales (Carnes, Hopkins, \& Green, 2014). Explorando la prevalencia del cuadro clínico, Ross, Mansson y Daneback (2012) encontraron que entre el 1.7\%-7.6\% de usuarios de Internet mostraba problemas en el control de su conducta sexual online. En nuestro país, el porcentaje de adolescentes que abusaba del cibersexo se situaba en el $8.6 \%$ en chicos y del 2.2\% en chicas (Ballester-Arnal, Giménez-García, Gil-Llario, \& Castro-Calvo, 2016); en adultos, el 90\% mostró un perfil de bajo riesgo de adicción al cibersexo, el $8.6 \%$ de riesgo medio y el $0.7 \%$ de alto riesgo (Ballester-Arnal, Castro-Calvo, Gil-Llario, et al., 2016).

Los estudios realizados hasta el momento exploran exhaustivamente la actividad sexual online problemática en adolescentes, jóvenes y adultos, pero desconocemos como este cuadro clínico se manifiesta en personas de mayor edad. La progresiva reducción de la brecha tecnológica y el incremento de la educación digital de los mayores ha aumentado el uso que éstos hacen de las TIC, lo que sumado a una vivencia cada vez más activa de su sexualidad offline (Lochlainn \& Kenny, 2013) y online (Castro-Calvo et al., 2017), les convierte en un colectivo vulnerable. Así, se planteó esta investigación a fin de: (1) analizar la prevalencia de mayores en riesgo de desarrollar o que ya muestran un perfil patológico de uso del cibersexo y (2) elaborar el perfil de signos y síntomas que lo caracterizan en esta población.

\section{MÉTODO}

\section{Participantes y procedimiento}

Los datos analizados en este trabajo se extrajeron de un estudio nacional sobre adicción al cibersexo realizado entre 2015 y 2018. Durante este periodo, aproximadamente 7.000 personas accedieron a una batería de autoinformes online en la que se evaluaba, entre otros muchos aspectos, las características del consumo de pornografía. El reclutamiento y la evaluación se realizó a través de ADISEX (http://adiccionalsexo.uji.es/), que es una plataforma diseñada ad hoc para la evaluación del control de los impulsos sexuales. Esta Web constituye un recurso abierto y accesible a través de cualquier motor de búsqueda mediante términos como "adicción al sexo", "adicción al cibersexo" 0 "tratamiento de la adicción al cibersexo". A fin de enriquecer el perfil de los participantes, se realizaron varias campañas mediante el sistema de publicaciones sugeridas de Facebook (sistema de márquetin de pago) y a través de otras vías de difusión formales (entrevistas en radio y televisión, listas de distribución, etc.) e informales (p.e., carteles informativos).

De los 7.000 evaluados, se seleccionó a 538 que superaban los 60 años (Rango=60-85; $M=65.34 ; D T=4.42)$. El $77 \%$ eran hombres y el $23 \%$ restante mujeres. La mayoría tenía pareja estable $(72.3 \%)$ y se autoidentificaba como heterosexual (92.7\%). En cuanto a las creencias religiosas, alrededor del $48.8 \%$ se definieron como ateos o agnósticos, seguido de creyentes no practicantes $(35.6 \%)$ y creyentes practicantes (15.6\%). La gran mayoría (99.1\%) dijo disponer de ordenador propio desde el que acceder a Internet. Finalmente, su distribución geográfica coincidía con las principales ciudades españolas en términos de densidad de población: es decir, el 17\% de los participantes decía residir en Madrid, el 10\% en Barcelona, el 6\% en Valencia y el 5\% en Sevilla.

\section{Instrumentos}

Los participantes completaron una batería online de evaluación compuesta por múltiples cuestionarios y escalas sobre el control de los impulsos sexuales online/ offline, así como otras variables psicológicas afines. El tiempo medio para completar la batería se situó en los 22.51 minutos $(S D=29.78)$. En este trabajo únicamente se analizaron los siguientes aspectos:

-Variables sociodemográficas: a través de un cuestionario $A d-H o c$, se preguntaba a los participantes acerca de su sexo, edad, provincia de residencia, si tenían una relación de pareja y de qué 
tipo (relación estable, relaciones esporádicas, etc.), orientación sexual, creencias religiosas y si disponían de ordenador propio con acceso a Internet.

-Comportamiento sexual online: se empleó el Cuestionario de Prácticas Sexuales Online (CPSO, Salusex-Unisexsida, 2016) para explorar aspectos como el tiempo dedicado a la actividad sexual online (“¿Cuántas horas a la semana pasas conectado a páginas sexuales?’), el tipo de actividades realizadas ("Contacto sexual por medio de Webcam", "Visionado de películas pornográficas"...) y la percepción de interferencia y gravedad (p.e., “¿Crees que pasas más tiempo del que deberías conectado a páginas sexuales en Internet?'). La fiabilidad de la escala de actividades sexuales online (única compuesta por varios ítems) fue de .83 (Alpha de Cronbach).

-Severidad del consumo de cibersexo: esta dimensión se evaluó mediante la adaptación española del Cuestionario de Adicción al Cibersexo (ISST, Ballester-Arnal, Gil-Llario, Gómez-Martínez, \& Gil-Julià, 2010). Compuesto por 25 ítems de respuesta dicotómica (Verdadero/Falso), este instrumento evalúa el grado en que la conducta sexual online resulta o no problemática. La suma de sus ítems permite obtener un índice general de adicción al cibersexo y clasificar a los participantes en 3 grupos (Carnes, Delmonico, \& Griffin, 2007): usuarios recreativos (0-8), de riesgo (9-18) y adictos (19-25). Ballester y cols. (2010) encontraron una fiabilidad de .88 para la escala general y una estabilidad temporal ( $r$ test-retest) de .82. En el presente estudio, la fiabilidad fue de .80 .

\section{Análisis de los datos}

Los datos se analizaron mediante el paquete estadístico SPSS Versión 23.0 y el software G*Power versión 3.1 (cálculo del tamaño del efecto). Se realizaron análisis descriptivos para caracterizar los participantes en términos sociodemográficos y de comportamiento sexual online, analizando posteriormente el papel del género en la modulación de dichas manifestaciones. Posteriormente, se emplearon varios criterios clínicos (p.e., puntuación en el ISST o autopercepción de interferencia) para clasificarlos en diferentes categorías, explorando nuevamente el papel del género en esta distribución. Una vez hecho esto, se analizó el perfil de signos y síntomas que mostraban los participantes clasificados en los grupos clínicos. Las diferencias en función del género se evaluaron mediante pruebas t de Student (para variables continuas) y contrastes Chi Cuadrado (variables categóricas). El tamaño del efecto en dichos contrastes se estimó a partir de la $d$ de Cohen y de la $V$ de Cramer (que en tablas Chi Cuadrado 2xk equivale al índice $W$ de Cohen del tamaño del efecto). Para la $d$ de Cohen, tamaños del efecto de alrededor de .20 se consideraron pequeños, cercanos a .50 moderados y superiores a .80 grandes (Cohen, 1988); en la $V$ de Cramer, estos tamaños correspondían a valores de $.10, .30$ y .50 respectivamente.

\section{RESULTADOS}

De los 538 participantes en esta investigación, el $73.2 \%$ afirmó haber utilizado Internet con fines sexuales. En hombres, el porcentaje superaba holgadamente el obtenido entre mujeres (83.3\% frente al 27\%), con diferencias que alcanzaban la máxima significación y un tamaño del efecto grande $\left(c h i^{2}=83.70 ; p<.001 ; V=.49\right)$. En adelante, Ios análisis se realizaron tomando únicamente a los participantes que sí habían practicado cibersexo.

A continuación, se clasificó a los participantes en diferentes categorías clínicas a partir de la puntuación total del ISST y los puntos de corte propuestos por Carnes et al. (2007) (tabla 1). De acuerdo con este criterio, ningún participante mostraba la severidad propia de un cuadro clínico de adicción al cibersexo, el 19.6\% encajaba en el perfil de usuarios de riesgo y la mayoría (el $80.4 \%$ ) lo hacía en el de uso recreativo. Todos los clasificados en el perfil de riesgo fueron hombres, alcanzado las diferencias en función del género la significación estadística $\left(C h{ }^{2}=13.8 ; p<.001\right)$ y un tamaño del efecto pequeño-moderado $(V=.22)$. 


\section{SIGNOS Y SÍNTOMAS DE ADICCIÓN AL CIBERSEXO EN ADULTOS MAYORES}

Tabla 1. Perfiles de consumo de cibersexo

\begin{tabular}{|c|c|c|c|c|c|}
\hline & Total & Hombres & Mujeres & $\mathrm{Chi}^{2}$ & $V$ \\
\hline Usuarios recreativos & $80.4 \%$ & $76.4 \%$ & $100 \%$ & & \\
\hline Usuarios de riesgo & $19.6 \%$ & $23.6 \%$ & $0 \%$ & $13.80 * * *$ & .22 \\
\hline Adictos al cibersexo & $0 \%$ & $0 \%$ & $0 \%$ & & \\
\hline
\end{tabular}

Nota: ${ }^{*} \mathrm{p}<0.05 ;{ }^{* *} \mathrm{p}<0.01 ;{ }^{* * *} \mathrm{p}<0.001$

Finalmente, se analizó el perfil de signos y síntomas de consumo excesivo de cibersexo de los participantes anteriormente clasificados como de riesgo (tabla 2). Habida cuenta de que en este perfil únicamente se incluyeron hombres, estos resultados no resultarían aplicables en mujeres. En primer lugar, se analizaron los síntomas psicopatológicos que incluye la subescala de comportamiento sexual compulsivo del ISST. Así, encontramos que el síntoma más frecuente (50.9\%) era pasar más de 5 horas a la semana realizando actividades sexuales online, seguido de la percepción de interferencia derivada de dicha actividad sexual (49.1\%) 0 del uso de Internet para experimentar con diferentes aspectos de la sexualidad (41.8\%). Dos aspectos íntimamente ligados con la expresión patológica de la conducta sexual online mostraron prevalencias un tanto inferiores: la presencia de síntomas de abstinencia (23.6\%) y la búsqueda del control activo del comportamiento a través del compromiso de abandonar la conducta (25.5\%) o bien castigarse si se realiza (10.9\%).

\section{Tabla 2. Signos y síntomas de consumo excesivo de cibersexo en participantes} clasificados como usuarios de riesgo

Prevalencia (\%)

Signos y síntomas de consumo excesivo de cibersexo según el ISST

Pasar $\geq 5$ horas semanales usando Internet con fines sexuales $50.9 \%$

Gastar más dinero del planeado durante la actividad sexual online

Percepción de que el sexo en Internet ha interferido con ciertos aspectos de la vida

Utilizar Internet para experimentar con diferentes aspectos de la sexualidad

Tener un sitio Web propio con contenido sexual $20 \%$

Utilizar cibersexo como premio por haber conseguido algo

Presencia de síntomas emocionales al no poder acceder a cibersexo (abstinencia)

Prometerse a uno mismo dejar de utilizar Internet con fines sexuales

Castigarse a uno mismo al utilizar Internet con fines sexuales

Otros signos y síntomas de consumo excesivo de cibersexo

$\geq 11$ horas semanales usando Internet con fines sexuales

$18.2 \%$

¿Crees que pasas más tiempo del que deberías conectado a páginas sexuales?

¿Te consideras adicto al sexo en Internet?

$40 \%$

Nota: ${ }^{*} \mathrm{p}<0.05 ; * * \mathrm{p}<0.01 ; * * * \mathrm{p}<0.001$ 
Asimismo, se analizaron otros indicadores con consumo excesivo de cibersexo. Cooper, Delmonico y Burg (2000) propusieron que el tiempo que alguien dedica en Internet con fines sexuales resultaría un indicador satisfactorio de consumo patológico. En concreto, proponían que las personas que pasaban $\geq 11$ horas a la semana en Internet mostraban más problemas e interferencia que las personas que invertían una cantidad de tiempo menor. Entre los usuarios de riesgo del presente estudio, sólo el 18.2\% cumplía con este criterio. Recientemente se ha propuesto que la autopercepción de consumo problemático constituiría otro indicador sensible en la detección de la adicción al cibersexo (Grubbs, Stauner, Exline, Pargament, \& Lindberg, 2015). En este sentido, se podrían establecer distintos niveles de percepción de gravedad en función de si la persona percibe que pasa demasiado tiempo realizando cibersexo (lo que no implicaría reconocer tácitamente que se padece patología alguna) o si se reconoce que puede estar padeciendo una adicción al cibersexo. Los resultados obtenidos a este nivel evidencian que el $50.9 \%$ de los usuarios de riesgo considera que pasa mucho tiempo en Internet con fines sexuales mientras que el $40 \%$ se consideraría adicto al cibersexo.

\section{DISCUSIÓN Y CONCLUSIONES}

El principal objetivo de esta investigación ha sido realizar una aproximación preliminar al estudio del comportamiento sexual online en un sector de población ignorado hasta el momento: los adultos mayores. En concreto, en este estudio se ha analizado el patrón de consumo excesivo y problemático de cibersexo de 538 personas de entre 60-85 años. La principal conclusión derivada de este trabajo es que el consumo patológico de cibersexo en este sector de población resulta prácticamente inexistente, su bien su abuso sí supera ampliamente al documentado hasta el momento en otras poblaciones en teoría expuestas a un riesgo mayor (por ejemplo, los jóvenes). Asimismo, muchos de los mayores que en teoría se ajustan a un perfil de riesgo, pero no patológico presentan síntomas definitorios de la adicción al cibersexo.

En cuanto a la prevalencia de usuarios patológicos de cibersexo, absolutamente ninguno de los participantes fue clasificado como tal pero un $19.6 \%$ (todos ellos hombres) lo fue como usuario de riesgo. Estos datos contrastarían con los obtenidos en estudios previos con jóvenes de entre 18-25 años, donde si bien el porcentaje de adictos al cibersexo era ligeramente mayor (del $0.7 \%$ ), el de usuarios de riesgo fue notablemente menor (8.6\%) (Ballester-Arnal, Castro-Calvo, Gil-Llario, et al., 2016). Considerando que los usuarios clásicamente agrupados en este perfil comparten la característica común de que el sexo online comienza a sobrevenir excesivo y a mostrar los primeros signos de interferencia (Cooper, 1998), este porcentaje subraya la importancia de analizar más a fondo el perfil de signos y síntomas presente en este grupo.

Cuando se analiza la presencia de diferentes signos y síntomas de adicción al cibersexo, se evidencia que un porcentaje importante de estos usuarios de riesgo (en algunos casos superior al $50 \%$ ) en realidad sí presentarían síntomas que encajan con un patrón de uso adictivo. Así, criterios tan característicos de este cuadro clínico como la abstinencia o la autopercepción de interferencia del comportamiento sexual online estarían presentes en un importante número de usuarios de riesgo que a priori, no alcanzarían una gravedad suficiente para el diagnóstico de esta condición. Cabría por tanto plantearse si tal vez los puntos de corte propuestos para el diagnóstico de la adicción al cibersexo mediante autoinforme (p.e., el ISST) se ajustarían a la realidad clínica de este colectivo, 0 bien en realidad estarían clasificando usuarios recreativos o de riesgo (en ambos casos, personas sin un patrón adictivo de consumo de cibersexo) a adultos mayores que en realidad sí presentarían síntomas de este cuadro clínico en un grado significativo. Habida cuenta del impacto sanitario de un falso negativo en el diagnóstico de un cuadro de adicción al cibersexo, se recomienda seguir investigando su manifestación en adultos mayores. 


\section{SIGNOS Y SÍNTOMAS DE ADICCIÓN AL CIBERSEXO EN ADULTOS MAYORES}

\section{REFERENCIAS BIBLIOGRÁFICAS}

Ballester-Arnal, R., Castro-Calvo, J., Gil-Llario, M. D., \& Gil-Julià, B. (2016). Cybersex Addiction: A Study on Spanish College Students. Journal of Sex \& Marital Therapy, 43(6), 567-584. https://doi.org/10.1080/0092623X.2016.1208700

Ballester-Arnal, R., Castro-Calvo, J., Gil-Llario, M. D., \& Giménez-García, C. (2014). Relationship status as an influence on cybersex activity: cybersex, youth, and steady partner. Journal of Sex \& Marital Therapy, 40(5), 444-456. https://doi.org/10.1080/0092623X.2013.772549

Ballester-Arnal, R., Gil-Llario, M. D., Gómez-Martínez, S., \& Gil-Julià, B. (2010). Psychometric properties of an instrument for assessing cyber-sex addiction. Psicothema, 22(4), 1048-1053.

Ballester-Arnal, R., Giménez-García, C., Gil-Llario, M. D., \& Castro-Calvo, J. (2016). Cybersex in the "Net generation": Online sexual activities among Spanish adolescents. Computers in Human Behavior, 57, 261-266. https://doi.org/10.1016/j.chb.2015.12.036

Carnes, P. J., Hopkins, T. A., \& Green, B. A. (2014). Clinical Relevance of the Proposed Sexual Addiction Diagnostic Criteria. Journal of Addiction Medicine, 8(6), 450-461. https://doi.org/10.1097/ADM.0000000000000080

Castro-Calvo, J., Ballester-Arnal, R., Gil-Llario, M. D., Giménez-García, C., \& Billieux, J. (2018). Online Sexual Activities (OSAs) in Spain: similarities and differences across the lifespan. In Abstracts of the 5th International Conference on Behavioral Addictions (p. 51).

Castro-Calvo, J., Ballester-Arnal, R., Giménez-García, C., \& Gil-Julià, B. (2017). Comportamiento sexual online en adultos mayores [Online Sexual Behavior in Older Adults]. International Journal of Developmental and Educational Psychology, 2(1), 89-98.

Castro-Calvo, J., Giménez-García, C., Gil-Llario, M. D., \& Ballester-Arnal, R. (2018). Motives to engage in Online Sexual Activities and their links to an excessive and problematic use: a Systematic Review. Current Addiction Reports, 1-20. https://doi.org/https://doi.org/10.1007/s40429-0180230-y

Cohen, J. (1988). Statistical Power Analysis for the Behavioral Sciences. Hillsdale, New Jersey: L. Erlbaum Associates.

Cooper, A. (1998). Sexually Compulsive Behavior. Contemporary Sexuality, 32(4), 1-3.

Cooper, A., Delmonico, D. L., \& Burg, R. (2000). Cybersex users, abusers, and compulsives: New findings and implications. Sexual Addiction \& Compulsivity, 7(1-2), 5-29. https://doi.org/10.1080/10720160008400205

Cooper, A., \& Griffin-Shelley, E. (2002). The internet:The next sexual revolution. In A. Cooper (Ed.), Sex \& the internet: A guidebook for clinicians (pp. 1-15). New York, NY: Brunner-Routledge.

Daneback, K., Cooper, A., \& Månsson, S.-A. (2005). An Internet Study of Cybersex Participants. Archives of Sexual Behavior, 34(3), 321-328. https://doi.org/10.1007/s10508-005-3120-z

Daneback, K., Sevcikova, A., Mänsson, S.-A., \& Ross, M. W. (2013). Outcomes of using the internet for sexual purposes: fulfilment of sexual desires. Sexual Health, 10(1), 26-31. https://doi.org/10.1071/SH11023

Goodson, P., McCormick, D., \& Evans, A. (2001). Searching for sexually explicit materials on the Internet: an exploratory study of college students' behavior and attitudes. Archives of Sexual Behavior, 30(2), 101-118.

Grubbs, J. B., Stauner, N., Exline, J. J., Pargament, K. I., \& Lindberg, M. J. (2015). Perceived Addiction to Internet Pornography and Psychological Distress: Examining Relationships Concurrently and over Time. Psychology of Addictive Behaviors, 29(4), 1056-1067. https://doi.org/10.1037/adb0000114

Kafka, M. P. (2013). The Development and Evolution of the Criteria for a Newly Proposed Diagnosis 
for DSM-5: Hypersexual Disorder. Sexual Addiction \& Compulsivity, 20(1-2), 19-26.

Kraus, S. W., Krueger, R. B., Briken, P., First, M. B., Stein, D. J., Kaplan, M. S., ... Reed, G. M. (2018). Compulsive sexual behaviour disorder in the Compulsive sexual behaviour disorder in the ICD11. World Psychiatry, 17(1), 109-110. https://doi.org/10.1002/wps.20499

Lochlainn, M. N., \& Kenny, R. A. (2013). Sexual activity and aging. Journal of the American Medical Directors Association, 14(8), 565-572. https://doi.org/10.1016/j.jamda.2013.01.022

Ross, M. W., Mansson, S. A., \& Daneback, K. (2012). Prevalence, severity and correlates of problematic sexual Internet use in Swedish men and women. Archives of Sexual Behavior, 51(2), 459-466. https://doi.org/10.1007/s10508-011-9762-0

Shaughnessy, K., \& Byers, E. S. (2014). Contextualizing cybersex experience: Heterosexually identified men and women's desire for and experiences with cybersex with three types of partners. Computers in Human Behavior, 32, 178-185. https://doi.org/10.1016/j.chb.2013.12.005

Shaughnessy, K., Byers, E. S., \& Walsh, L. (2011). Online Sexual Activity Experience of Heterosexual Students: Gender Similarities and Differences. Archives of Sexual Behavior, 40(2), 419-427.

Smith, M. (2013). Youth Viewing Sexually Explicit Material Online: Addressing the Elephant on the Screen. Sexuality Research and Social Policy, 10(1), 62-75. https://doi.org/10.1007/s13178012-0103-4

Wéry, A., \& Billieux, J. (2017). Problematic cybersex: Conceptualization, assessment, and treatment. Addictive Behaviors, 64, 238-246. https://doi.org/10.1016/j.addbeh.2015.11.007

Wolak, J., Mitchell, K., \& Finkelhor, D. (2007). Unwanted and Wanted Exposure to Online Pornography in a National Sample of Youth Internet Users. PEDIATRICS, 119(2), 247-257. https://doi.org/10.1542/peds.2006-1891 
